\title{
GM1 controlled lateral segregation of tyrosine kinase Lck predispose T-cells to cell-derived galectin-1-induced apoptosis
}

\author{
Julianna Novák ${ }^{a}$, Éva Kriston-Pála , Ágnes Czibulaa ${ }^{a}$ Magdolna Deák ${ }^{b}$, László Kovács ${ }^{\text {b }}$, \\ Éva Monostori ${ }^{a}$, Roberta Fajka-Boja ${ }^{a, *}$
}

a Institute of Genetics, Biological Research Centre, Hungarian Academy of Sciences, H-6726 Szeged, Hungary

b Department of Rheumatology, Albert Szent-Györgyi Health Centre, University of Szeged, H-6726 Szeged, Hungary

\section{A R T I C L E I N F O}

\section{Article history:}

Received 6 June 2013

Received in revised form 9 September 2013

Accepted 15 October 2013

\section{Keywords:}

Galectin-1

Lck

CD45

GM1

Apoptosis

\begin{abstract}
A B S T R A C T
One prominent immunoregulatory function of galectin-1 (Gal-1), a $\beta$-galactoside binding mammalian lectin, is induction of apoptosis in activated T-cells by a process depending on the activity of Src family tyrosine kinase, Lck. Although the requirement for Lck in Gal-1 induced T-cell death and the ability of Gal-1 to affect the membrane localization of extracellular Gal-1-binding proteins have been well documented, the consequence of the complex and related reorganization of extra- and intracellular signaling components upon Gal-1 treatment of T-cells has not yet been revealed. Therefore, we have analyzed the plasma membrane movement of Lck upon Gal-1 triggered signaling, and the significance of this event in Gal-1 induced T-cell death. Non-receptor tyrosine kinase, Lck primarily localized in the synapse of tumor cell-T-cell during $15 \mathrm{~min}$ of the established direct cell contact. Later, after $30 \mathrm{~min}$, a lateral segregation of Lck from the cell synapse was observed. The migration of Lck to the opposite of the cell contact apparently depended on the expression and cell surface presentation of Gal-1 on the effector (tumor) cells and was accompanied by phosphorylation on the negative regulatory tyrosine residue, Tyr 505 . Receptor tyrosine phosphatase, CD45 played crucial role in this event since CD45 deficiency or inhibition of its phosphatase activity resulted in the failure of Lck membrane movement. Level of the Gal-1-binding glycolipid GM1 ganglioside also essentially regulated Lck localization. Segregation of Lck and Gal-1 induced apoptosis was diminished in T-cells with low GM1 expression compared to T-cells with high GM1. Our results show that spatial regulation of Lck by CD45 and GM1 ganglioside determines the outcome of apoptotic response to Gal-1 and this local regulation may occur only upon intimate effector (Gal-1 expressing) cell-T-cell attachment.
\end{abstract}

c 2013 Elsevier Ltd. All rights reserved.

\section{Introduction}

T-cells and antigen presenting cells form immunological synapses, which are considered to assemble from specific membrane microdomains, rafts (Bromley et al., 2001; Dykstra et al., 2001 ), composed of T-cell receptor (TCR), co-receptors and adhesion molecules in a specific lipid environment, and scaffolded by the actin cytoskeleton (Burkhardt et al., 2008). The composition of membrane rafts varies during different differentiation and activation phases of T-cells, e.g. the amount of GM1 ganglioside

Abbreviations: Gal-1, galectin-1; TCR, T-cell receptor; HeLamock, mock transfected HeLa cells; HeLa ${ }^{\text {Gal-1 }}$, Gal-1 transfected HeLa cells; $\beta$-CD, $\beta$-cyclodextrin; CTX-FITC, cholera toxin B subunit - fluorescein isothiocyanate.

* Corresponding author at: Biological Research Centre of Hungarian Academy of Sciences, Temesvári kıt 62, H-6726 Szeged, Hungary. Tel.: +36 62599600 ; fax: +3662433503 .

E-mail address: fajka_boja.roberta@brc.mta.hu (R. Fajka-Boja). is expressed at higher levels in effector cells, compared to resting T-cells (Tuosto et al., 2001). Regulation of GM1 expression is substantial, as alteration in lipid raft composition may lead to inappropriate $\mathrm{T}$ lymphocyte signaling and ultimately to development of pathological conditions (Jury et al,, 2004). In resting T-cells, TCR and tyrosine kinases Lck and ZAP-70 are excluded from or weakly associated with rafts, however, they translocate into raft domains after TCR engagement (Montixi et al., 1998). Localization of activated Lck to rafts is important for the initial signaling which drives subsequent activation of signaling proteins, cytoskeletal changes, membrane remodeling and formation of stable signaling complexes at TCR activation sites (Salmond et al., 2009). Activity of Lck is regulated by phosphorylation of two conserved tyrosine residues, Tyr505 and Tyr394. Phosphorylation at Tyr505 results in a closed, enzymatically inactive conformation. When outside of the rafts, Lck is dephosphorylated by $C D 45$ receptor tyrosine phosphatase at the Tyr-505, thereby it is potentiated for activation and autophosphorylation at the Tyr394 (Sieh et al., 1993; Rodgers and Rose, 1996). In turn, when the active Lck is recruited to adaptor $\mathrm{Cbp} /$ PAG in rafts, 
Csk kinase phosphorylates the negative regulatory Tyr505 of Lck (Kawabuchi et al., 2000) resulting in inactivation, hence terminating the activation process.

TCR clustering at the immune synapse is negatively regulated by particular members of the galectin family, $\beta$-galactoside binding lectins, which interact with cell surface glycoproteins and glycolipids and form lattice, thereby restricting glycoprotein movement in the cell membrane and inhibiting reorganization of signaling complexes (Grigorian et al., 2009). Accordingly, soluble Gal-1 binding to T-cells induces reorganization of Gal-1 binding receptors, and as a result CD45 and CD3 are co-localized on large islands of apoptotic blebs and CD7 and CD43 are co-localized in small patches (Pace et al., 1999). Specific membrane microdomain structure is crucial during Gal-1 induced T-cell apoptosis, since disruption of rafts results in failure of execution of Gal-1 signaling and induction of cell-death (Ion et al., 2006; Kovács-Sólyom et al., 2010). This apoptotic process is characterized by early tyrosine phosphorylation with involvement of tyrosine kinase, Lck, followed by ceramide production by acid sphingomyelinase, mitochondrial depolarization, caspase- 9 and -3 activation (Ion et al., 2005, 2006).

Gal-1 is often upregulated in tumor cells and the surrounding stroma and it is supposed to contribute to tumor immune privilege (Rubinstein et al,, 2004). We have recently shown that the Gal-1 produced by and bound to the surface of tumor cells induces cell death in Jurkat cells or PHA activated human peripheral blood Tcells in an ex vivo co-culture system (Kovács-Sólyom et al., 2010). This system resembles better the in vivo milieu than usage of soluble protein, as lymphocytes encounter Gal-1 attached to the cell surface of the producer cells or components of the extracellular matrix (He and Baum, 2004). In this work we analyze the plasma membrane movements of Lck, a major component of Gal-1 triggered apoptotic signaling. We show that the membrane localization of Lck is affected by CD45, GM1 ganglioside and intact raft organization. Most importantly, we provide a novel insight into those membrane proximal events which eventually lead to T-cell apoptosis.

\section{Materials and methods}

\subsection{Cells}

All cell lines were kept in an incubator with $5 \% \mathrm{CO}_{2}$ at $37^{\circ} \mathrm{C}$. Jurkat cell lines and the CD45 deficient Jurkat variant, J45.01 (European Collection of Cell Cultures) were cultured in RPMI1640 (Gibco, Invitrogen) supplemented with penicillin (100 IU/ml), streptomycin $(100 \mu \mathrm{g} / \mathrm{ml})$, L-glutamine $(2 \mathrm{mM})$ and in the presence of $5 \%$ (Jurkat) or $10 \%$ (J45.01) heat inactivated fetal calf serum (FCS) (Gibco, Invitrogen). C32 human melanoma cells and mock transfected (HeLamock) or Gal-1 transgenic (HeLa ${ }^{\text {Gal-1}}$ ) human cervix adenocarcinoma cells were cultured in MEM (Gibco, Invitrogen) supplemented with penicillin, streptomycin, L-glutamine and 5\% (C32) or $10 \%$ (HeLa) FCS. Peripheral blood mononuclear (PBM) cells from healthy human donors were isolated through Ficoll gradient (GE Healthcare) and activated with $5 \mu \mathrm{g} / \mathrm{ml}$ phytohemagglutininM (PHA, Calbiochem) for $72 \mathrm{~h}$ in RPMI containing $10 \%$ FCS. Studies involving human blood samples were conducted in accordance with the guidelines of the Declaration of Helsinki and have been approved by the institutional ethics committee of the University of Szeged.

To separate cells based on their GM1 ganglioside expression Jurkat or human activated $\mathrm{T}$-cells were washed and resuspended in DMEM at $10^{7}$ cells $/ \mathrm{ml}$, and then $20 \mu \mathrm{g} / \mathrm{ml}$ cholera toxin B subunit (CTX)-biotin (Sigma) was added. After $15 \mathrm{~min}$ incubation on ice the cells were washed with DMEM and $50 \mu$ BD IMag $^{\text {TM }}$ Streptavidin Particles Plus - DM (BD Bioscience) was added to $10^{7}$ cells for $30 \mathrm{~min}$ at $4^{\circ} \mathrm{C}$. The cell concentration was set to $10^{7}$ cells $/ \mathrm{ml}$ and the tube was placed into the magnet (BD IMaq ${ }^{\mathrm{TM}}$ cell separation magnet) for $8 \mathrm{~min}$. The negative fraction (supernatant) and the positive fraction (pellet) were separately cultured, and tested for the expression of GM1 by flow cytometry. Cells from negative fraction expressing low level of GM1 were designated as J.GM1 ${ }^{\text {lo }}$ and GM1 ${ }^{\text {lo }}$ T-cells for Jurkat and human activated T-cells, respectively. Activated T-cells with high GM1 expression were referred as GM1 ${ }^{\text {hi }}$ T-cells. J.GM1 ${ }^{\text {lo }}$ cells were maintained in RPMI 1640 with 5\% FCS and tested for their GM1 expression before use by flow cytometry. Separated human T-cells were used immediately for apoptosis tests.

\subsection{Flow cytometry}

GM1 detection. The cells were washed in ice cold phosphate buffered saline (PBS) supplemented with $1 \%$ FCS and $0.1 \%$ sodiumazide (FACS-buffer) and resuspended at $2 \times 10^{6}$ cells $/ \mathrm{ml}$, and then CTX-FITC was added at a concentration of $10 \mu \mathrm{g} / \mathrm{ml}$. After incubation for 30 min on ice the samples were washed with FACS-buffer, and measured on FACSCalibur flow cytometer using CellQuest ${ }^{\mathrm{TM}}$ software (Becton \& Dickinson).

Detection of surface Gal-1. HeLa ${ }^{\text {mock }}$ cells were treated with $50 \mu \mathrm{g} / \mathrm{ml}$ recombinant Gal-1 (rGal-1), then unbound lectin was removed with washing with FACS-buffer. Amount of surface Gal-1 on Gal-1-treated HeLa ${ }^{\text {mock }}$ and transgenic HeLa ${ }^{\text {Gal-1 }}$ cells was analyzed by FITC conjugated anti-Gal- $1 \mathrm{mAb}$ (clone $2 \mathrm{c} 1 / 6$, produced in our laboratory, Kovács-Sólyom et al., 2010) and cytofluorimetry was carried out as described above.

\subsection{Co-cultures of T-cells and Gal-1 producing tumor cells}

C32 ( $10^{4}$ cells/sample) or HeLamock and $\mathrm{HeLa}^{\text {Gal- } 1}$ cells $\left(5 \times 10^{3}\right.$ cells/sample) were plated onto round cover slips $(12 \mathrm{~mm}$ diameter, Menzel Gläser, Thermo Scientific) in 24-well plate. Jurkat, J.GM1 $1^{\mathrm{lo}}$, J45.01 or GM1 $1^{\mathrm{lo}}$ and GM1 $1^{\text {hi }}$ activated human T-cells $\left(2-5 \times 10^{5}\right.$ cells/sample) were labeled with Hoechst 33342 $\left(100 \mathrm{ng} / \mathrm{ml}\right.$ for $30 \mathrm{~min}$ at $37^{\circ} \mathrm{C}$ ) and co-cultured with tumor cells for the indicated time points. In particular experiments Jurkat cells were incubated with the following inhibitors before added to tumor cells: $\beta$-cyclodextrin ( $\beta$-CD) for raft disruption $(10 \mathrm{mM}$, $30 \mathrm{~min}$, Sigma) or PTP CD45 inhibitor $(10 \mu \mathrm{M}, 20 \mathrm{~min}$, Santa Cruz Biotechnology). These inhibitors were present throughout the experiments.

For competing galectin binding to cell surface HeLamock and HeLa ${ }^{\text {Gal- }} 1$ cells were incubated with $100 \mathrm{mM}$ lactose (Sigma) for $30 \mathrm{~min}$ at $4{ }^{\circ} \mathrm{C}$ in cell culture medium, and then galectins were removed with extensive washing before co-culture. $50 \mu \mathrm{g} / \mathrm{ml}$ human recombinant Gal-1 was added to indicated samples of lactose washed HeLa ${ }^{\text {mock }}$ cells for 30 min at $4{ }^{\circ} \mathrm{C}$ and then the unbound Gal-1 was removed with washing before co-culture.

\subsection{Immunocytochemistry}

T-cells and tumor cells were co-cultured for the indicated time points (ranging from $15 \mathrm{~min}$ to $1 \mathrm{~h}$ ), then fixed in $4 \%$ paraformaldehyde for $4 \mathrm{~min}$ at room temperature, washed in PBS and the cover slips were saturated in FACS-buffer for $1 \mathrm{~h}$ prior immunostaining. The following antibodies were used: unlabeled or FITC conjugated mouse anti-Lck mAb (produced in our laboratory, Ion et al., 2005), PE conjugated mouse anti-LCK pTyr505 (BD ${ }^{\mathrm{TM}}$ Phosflow), 4G10 anti-phosphotyrosine mAb (Upstate Biotechnology, Millipore), unlabeled or FITC conjugated anti-Gal-1 mAb (clone 2c1/6, produced in our laboratory, Kovács-Sólyom et al., 2010). Cell surface staining was carried out in FACS-buffer. For intracellular staining the antibodies were added to the cells in FACS-buffer 
containing $75 \mu \mathrm{g} / \mathrm{ml} \mathrm{L}-(\alpha)$-lysophosphatidylcholine (LPC, Sigma), that rapidly permeabilize the cell membrane while retains cells' integrity (Chitu et al., 1999). Unlabeled primary antibodies were followed by anti-mouse IgG-NorthernLights-557 (R\&D Systems). To visualize the cell-cell contact, F-actin was labeled with RhodaminePhalloidin (Invitrogen). The cover slips were mounted on slides with a drop of Fluoromount-G (SouthernBiotech). The samples were analyzed with Axioskop 2Mot (Carl Zeiss) fluorescence microscope using $40 \times$ objective magnification, or confocal images were taken with Olympus FV 1000 laser scanning confocal microscope, $60 \times$ objective and zoom function. The correct exposure was determined based on the intensity plots generated by FV10-ASW 2.0 Viewer software (Olympus) (Supplementary Fig. 1). Photos were taken from 10 randomly selected non-overlapping fields of the samples, and at least 100 cells/sample were analyzed. Localization of Lck in the cell-cell contact or in membrane domains opposite to cell-cell contact (sequestered) were determined from non-adjusted raw pictures and evaluated by the following equation: percentage of cells with the Lck sequestered or in cell contact = number of Tcells with Lck sequestered or in cell contact/number of T-cells in contact with tumor cells) $\times 100$. To determine the relative effect of Gal-1, the percentages of cells with sequestered Lck in samples with $\mathrm{HeLa}^{\text {mock }}$ were subtracted from results with $\mathrm{HeLa}^{\mathrm{Gal}-1}$. For presentation of the images, the contrast of the images was adjusted using Adobe Photoshop CS4 extended 11.0 (Supplementary Fig. 1).

\subsection{Detection of apoptosis in co-culture system}

After $16 \mathrm{~h}$ of co-culture the cells were fixed in $4 \%$ paraformaldehyde for $4 \mathrm{~min}$ at room temperature and labeled for phosphatidylserine exposure on the outer cell membrane as described previously (Kovấcs-Sólyom et al., 2010). The samples were analyzed with Axioskop 2Mot (Carl Zeiss) fluorescence microscope using AxioCam camera, AxioVision 3.1 software and $20 \times$ objective magnification. The contrasts of the images were adjusted using Adobe Photoshop CS4 extended 11.0. At least 100 cells/sample were analyzed and the rate of apoptosis was determined as follows: \% of apoptotic cells $=($ Annexin $V$ positive cells $/$ total cell number $) \times 100$.

The active (cleaved) form of caspase- 3 was detected after $24 \mathrm{~h}$ of T-cell/tumor cell co-culture. The cells were fixed in $4 \%$ paraformaldehyde for $25 \mathrm{~min}$ at room temperature and permeabilized with $0.1 \%$ Triton X-100 in FACS-buffer for 10 min, then saturated in FACS-buffer for $1 \mathrm{~h}$. The samples were reacted with rabbit anti-caspase-3 (Cell Signaling Technology) and anti- rabbit Ig-FITC (Sigma). After washing the cover slips were mounted on slides with a drop of Fluoromount-G and analyzed with Axioskop 2Mot fluorescence microscope.

\subsection{Statistics}

Average and standard deviation were determined with Microsoft EXCEL software from the results of 3-5 independent samples, as indicated in figure legends. Statistical significance of the differences was determined using the Student's t test (set at $\left.{ }^{*} p<0.05,{ }^{* *} p<0.01,{ }^{* * *} p<0.001\right)$.

\section{Results}

\subsection{Cell-derived galectin-1 induces exclusion of Lck from cell synapse}

We have recently shown that stimulation of T-cells with soluble recombinant Gal-1 induced remarkable tyrosine phosphorylation with the involvement of Lck non-receptor tyrosine kinase leading eventually to apoptosis (Ion et al., 2005, 2006). Lack of Lck in
Jurkat derivative cell line, J.CaM1.6 rendered these T-cells resistant to Gal-1 induced cell death (Ion et al., 2006). Similarly, tumor cell-derived Gal-1 also induced T-cell death on an Lck dependent fashion (Kovács-Sólyom et al., 2010). The central role of Lck in Gal1 triggered T-cell death motivated the analysis of early membrane remodeling, an event determining the final signaling outcome, with a special focus on Lck redistribution in T-cells upon Gal-1 stimulation, We applied a co-culture system using Gal-1 non-producing (HeLa ${ }^{\text {mock }}$ ) or Gal-1 transgenic ( HeLa ${ }^{\text {Gal-1 }}$ ) HeLa cell lines as effector cells (for characterization see Supplementary Fig. 2, and KovácsSólyom et al., 2010). As we showed earlier the ultimate requirement of HeLa ${ }^{\text {Gal-1 }}$ triggered T-cell apoptosis was the direct cell-cell contact (Kovács-Sólyom et al., 2010).

T-cells and HeLa cells formed intimate cell-cell interaction within $15 \mathrm{~min}$ of co-culture, and as a result, the membrane associated Gal-1 rapidly transferred from the surface of HeLaGal-1 to Jurkat cells (Fig. 1A right panel). Gal-1 was not detectable on Jurkat co-cultured with HeLamock (Fig. 1A left panel). This result was in accordance to our previous finding (Kovács-Sólyom et al., 2010), and was reproducible when using another Gal-1 producing cell line, the C32 human melanoma (Supplementary Fig. 3). At early time point, $15 \mathrm{~min}$ of the cell-cell interaction, Gal-1 was mainly accumulated in cell contact (Fig. 1A) and then dispersed evenly on the cell surface of Jurkat (data not shown). Tight cell synapse between the tumor cell and T-cell, as detected by actin staining (Fig. 1B), generated different distribution of tyrosine kinase Lck depending on the absence or presence of Gal-1 in the effector cells: Lck clustered to the cell synapse of Jurkat and HeLamock (Fig. 1B, upper panel) while it was sequestered to distal membrane site when Jurkat cells adhered to HeLa ${ }^{\text {Gal-1 }}{ }^{\text {(Fig. } 1 \mathrm{~B} \text {, lower panel) after } 60 \mathrm{~min}}$ of co-culture. Localization of Lck to the opposite of the cell contact was significantly more frequent in cell-cell contact with HeLaGal-1 than HeLa ${ }^{\text {mock }}$ cells (Fig. 1C and D), indicating the role of Gal-1 in this process. Plasma membrane distribution of Lck was uniform in untreated Jurkat cells (Supplementary Fig. 4). Lck appeared at the cell synaptic site when Jurkat cells were co-cultured with $\mathrm{HeLa}^{\text {mock }}$ and kept this position for up to $60 \mathrm{~min}$ (Fig. 1E). In contrast, a gradual, time dependent accumulation of Lck distal from the cell synapse was observed when HeLaGal-1 and Jurkat cells established cell contact. (Fig. 1E).

Specific role of Gal-1 in Lck membrane movement from cell synapse was examined in various experimental designs. First, $\mathrm{HeLa}^{\text {mock }}$ or lactose (competitor of galectins)-washed HeLamock induced Lck sequestration only in minor percentage of T-cells. In contrast, HeLamock cells covered with rGal-1induced high Lck sequestration (Fig. 1D lac HeLamock + rGal-1). Second, removal of surface Gal-1 from HeLa ${ }^{\text {Gal-1 }}$ with lactose (Fig. 1D lac HeLaGal-1) diminished the Lck exclusion from cell synapse. Lck sequestration was higher when induced by rGal-1 treated HeLamock than by transgenic HeLaGal-1 since surface Gal-1 amount was higher on $\mathrm{HeLa}{ }^{\text {mock }}+\mathrm{rGal}^{-1}$ than on HeLaGal-1 (Supplementary Fig. 2B and $\mathrm{C}$ ). To rule out the effect of other galectins, all cell-surface $\beta$ galactoside binding lectins were removed from HeLa ${ }^{\text {mock }}$ (Fig. 1D lac HeLa ${ }^{\text {mock }}$ ) surface with lactose, however, it had no effect compared to non-treated HeLamock indicating that the directed Lck membrane movement was not due to other types of galectins (Fig. 1D HeLa ${ }^{\text {mock }}$ vs. lac HeLa ${ }^{\text {mock }}$ ). Moreover, analysis of gene expressions of Gal-1, Gal-3 (Supplementary Fig. 5) and other galectins, such as Gal-7 and -8 (data not shown) showed that $\mathrm{HeLa}^{\text {mock }}$ did not express none of these galectins while HeLaGal-1 expressed only Gal-1.

The Gal-1 induced Lck sequestration was also detected when Jurkat cells were co-cultured with C32 melanoma (Supplementary Fig. 6), showing that other Gal-1 secreting cells are also capable to trigger this process. To ensure that the altered glycosylation in malignant T-cells (Ju et al,, 2011) did not affect the examined cell 
A
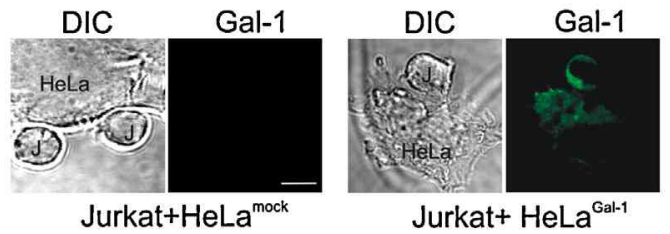

B
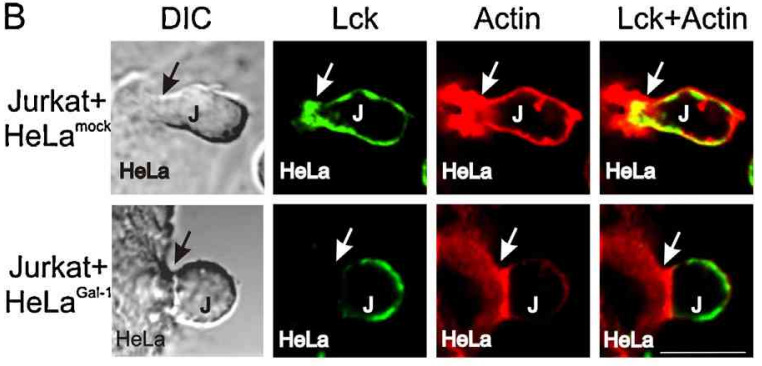

C

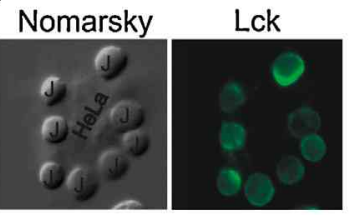

Jurkat+HeLa ${ }^{\text {mock }}$

D

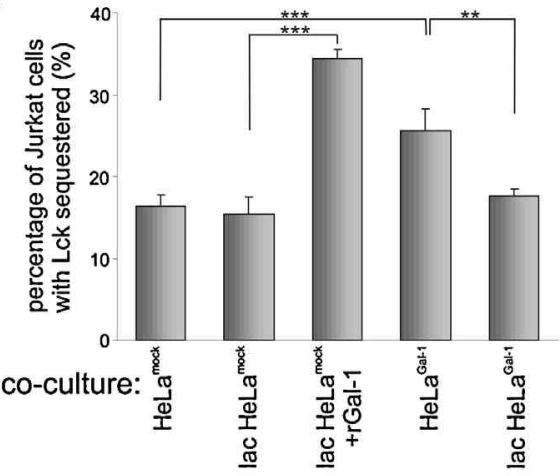

E

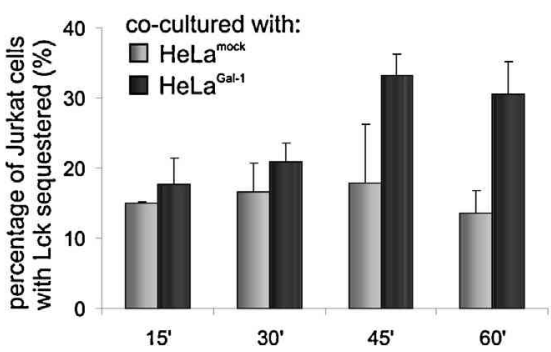

Fig. 1. Cell-derived Gal-1 induced sequestration of Lck from cell synapse in Jurkat cells. Jurkat (J) cells were co-cultured with Gal-1 negative (HeLa ${ }^{\text {mock }}$ ) or Gal-1 producing tumor cells (HeLa ${ }^{\mathrm{Gal}-1}$ ) for different time points $(15 \mathrm{~min}$ for $\mathrm{A}, 1 \mathrm{~h}$ for $\mathrm{B}-\mathrm{D}$, 15-60 min for E), and then the cells were fixed. (A) Cell surface bound Gal-1 was detected with anti-Gal-1-FITC in confocal microscope. (B and C) In permeabilized cells Lck and F-actin were labeled with FITC conjugated anti-Lck (green, B and C) and rhodamine-phalloidin (red, B) respectively, and detected with confocal (B) or fluorescence microscopy (C). Arrows indicate the cell synapse. (D) Jurkat cells were in co-culture with HeLa ${ }^{\text {mock }}$, lactose pre-treated HeLamock (lac Helamock $^{\text {) lactose pre- }}$ treated HeLamock with recombinant Gal-1 bound (lac Helamock + rGal-1), HeLa ${ }^{\text {Gal-1 }}$ or lactose pre-treated HeLa ${ }^{\text {Gal-1 }}$ (lac Hela ${ }^{\text {Gal-1 }}$ ) for $1 \mathrm{~h}$. The cells were then fixed, permeabilized and labeled with mouse anti-Lck-FITC and analyzed in fluorescence microscope. The percentage of Jurkat cells with sequestered Lck was calculated as described in Section 2.4. The average and standard deviation was calculated from 3 to 4 samples. Student's $t$-test, ${ }^{* *} p<0.01,{ }^{* * *} p<0.001$. (E) The percent age of Jurkat cells with sequestered Lck was determined for each time points (15-60 min) of co-culture and results from 2 independent experiments were presented. (For interpretation of the references to color in text, the reader is referred to the web version of this article.)

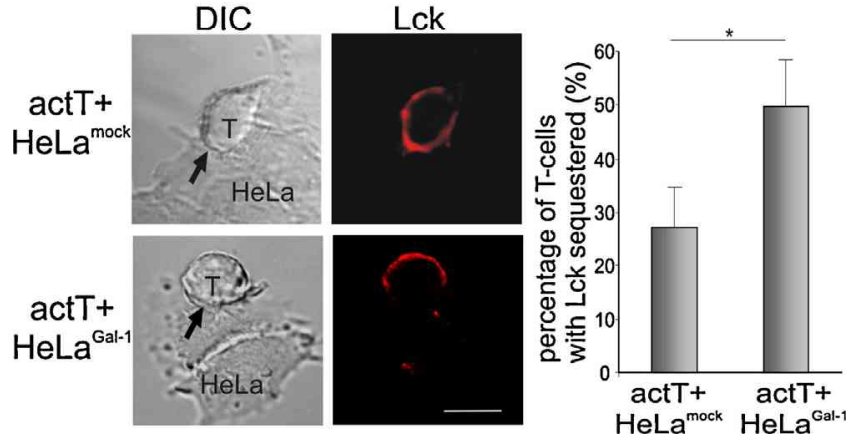

Fig. 2. Cell-derived Gal-1 induced sequestration of Lck from cell synapse in PHA activated human T-cells. PBMC were activated with PHA for 3 days (actT) and then co-cultured with HeLamock or HeLa ${ }^{\text {Gal-1 }}$ for $1 \mathrm{~h}$. The cells were then fixed, permeabilized and labeled with mouse anti-Lck and anti-mouse IgG-NL557 (red) and analyzed with confocal microscope. Arrows indicate the cell synapse. Scale bar: $10 \mu \mathrm{m}$. The percentage of T-cells with sequestered Lck was determined as described in Section 2.4. The average and standard deviation was calculated from 4 samples. Student's $t$-test, ${ }^{*} p<0.05$. (For interpretation of the references to color in text, the reader is referred to the web version of this article.)

A
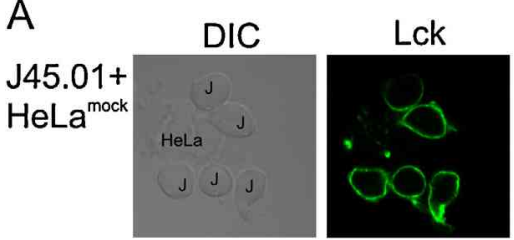

B
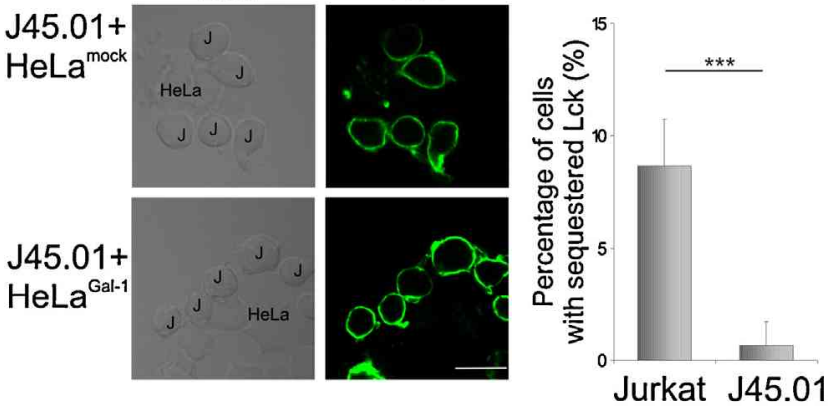

C
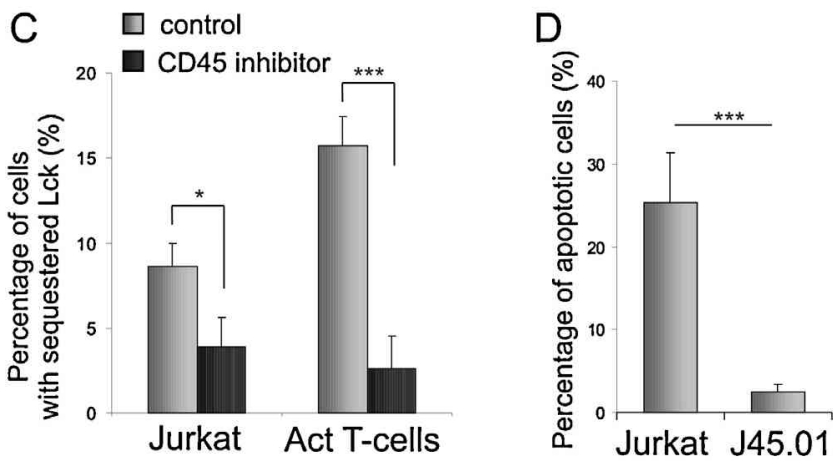

Fig. 3. Activity of CD45 phosphatase was required for Lck sequestration. (A) CD45 deficient $\mathrm{J45.01}$ cells were co-cultured with $\mathrm{HeLa}^{\mathrm{Gal}-1}$ or HeLamock for $1 \mathrm{~h}$, then fixed and permeabilized. Lck was detected with mouse anti-Lck-FITC (green) by confocal microscopy. Scale bar: $10 \mu \mathrm{m}$. (B) Sequestration of Lck in Jurkat and the CD45 deficient J45.01 cells was compared in the co-culture with HeLa ${ }^{\mathrm{Gal}-1}$ or He La ${ }^{\mathrm{mock}}$ for $1 \mathrm{~h}$. The average and standard deviation of the percentage of cells with sequestered Lck was calculated from 4 samples of two independent experiments, and the subtraction of the values obtained in co-culture with $\mathrm{HeLa}^{\mathrm{Gal}-1}$ and HeLamock was presented, as described in Section 2.4. (C) Jurkat cells and PHA-activated T-cells were pre-treated with PTP CD45 inhibitor ( $10 \mu \mathrm{M}$ for $20 \mathrm{~min}$ ) and then co-cultured with HeLa ${ }^{\mathrm{Gal}-1}$ or $\mathrm{HeLa}^{\text {mock }}$ for $1 \mathrm{~h}$. Sequestration of Lck was determined from 4 samples of two independent experiments as described in previous point. (D) Jurkat and J45.01 cells were co-cultured with $\mathrm{HeLa}^{\mathrm{Gal}-1}$ or HeLa ${ }^{\text {mock }}$ for $16 \mathrm{~h}$ and then reacted with Annexin $\mathrm{V}$ AlexaFluor 488. Apoptosis was detected by fluorescent microscopy, and calculated as described in Section 2.5. Student's $t$-test, ${ }^{*} p<0.05,{ }^{* * * *} p<0.001$. (For interpretation of the references to color in text, the reader is referred to the web version of this article.) 
response, we verified our findings using human PHA-activated Tcells (actT). Similarly to Jurkat cells, significantly higher percentage of sequestered Lck was detected in actT when co-cultured with $\mathrm{HeLa}^{\mathrm{Gal}-1}$ as with HeLa ${ }^{\text {mock }}$ (Fig. 2). These results show that Gal-1 plays critical role in the spatial regulation of Lck upon encounter of tumor cells and T-cells.

\subsection{Involvement of $C D 45$}

The receptor tyrosine phosphatase, CD45 has been implicated as the main regulator of Lck activity and importantly it has been identified as a major Gal-1 binding protein (Pace et al., 1999; Walzel et al., 1999; Fouillit et al., 2000; Fajka-Boja et al., 2002). Whether CD45 plays a role in Gal-1 induced Lck segregation has been examined. As Fig. 3 shows CD 45 deficient variant of Jurkat, J45.01, readily attached to HeLa cells but did not respond with Lck sequestration, irrespectively of Gal-1 production by HeLa cells (Fig. 3A and B). This finding was further supported by using CD 45 specific PTP inhibitor, since Lck segregation failed in the presence of the inhibitor either in Jurkat or actT-cells (Fig. 3C). Hence, both the presence and the phosphatase activity of CD 45 were necessary in Gal-1 triggered Lck segregation. Effect of CD45 was not limited to Lck relocalization since J45.01 cells were unable to respond to HeLaGal-1 with apoptosis (Fig. 3D). These findings supported that the early membrane movements are important in the downstream cell death process.

\subsection{Kinetics of P-Tyr505 Lck exclusion from cell synapse}

Kinase activity of Lck is negatively regulated by the tyrosine phosphorylation of its Tyr505 residue. Indeed, Lck species phosphorylated on this particular tyrosine were excluded from the synaptic zone in a time dependent manner when Jurkat cells were co-cultured with HeLaGal-1 (Fig. 4A). The localization and kinetics of sequestration of inactive P-Tyr505 Lck coincided with that of the total Lck pool (Fig. 4B and Fig. 1E, respectively). As expected, the signaling cascade initiated by Lck resulted in accumulation of tyrosine phosphorylated proteins in the synaptic zone (Fig. 4C and D). It has to be noted that HeLamock did not trigger specific membrane localization of Lck, P-Tyr505 Lck or tyrosine phosphorylated proteins (Fig. 4B and D).

\subsection{GM1 ganglioside affects Gal-1 induced Lck segregation and apoptosis}

One of the major Gal-1-binding cell surface glycoconjugates is the GM1 ganglioside (Wang et al., 2009; Fajka-Boja et al., 2008). Moreover, GM1 is a regular lipid component of rafts, which are the platform of signaling complexes (Tani-ichi et al., 2005). We verified the importance of raft formation in Gal-1-induced apoptosis, since disrupting the integrity of membrane microdomains with $\beta$-cyclodextrin $(\beta-C D)$, a cholesterol-chelator, inhibited the
A

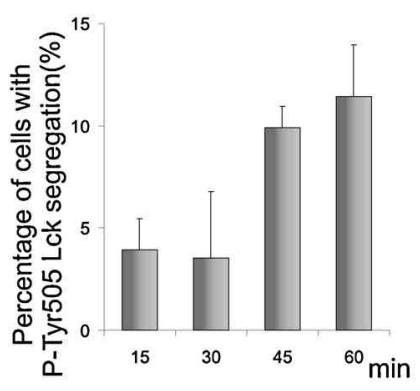

C

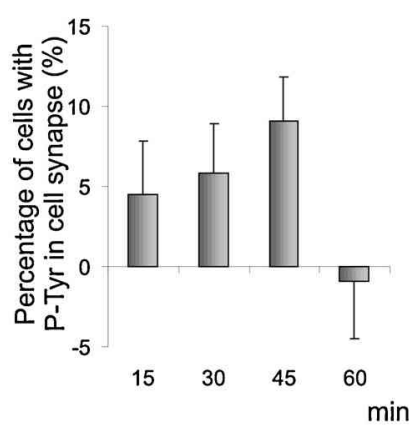

B

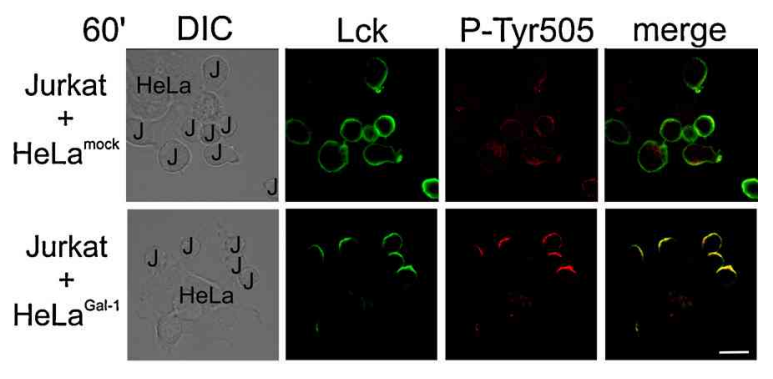

D

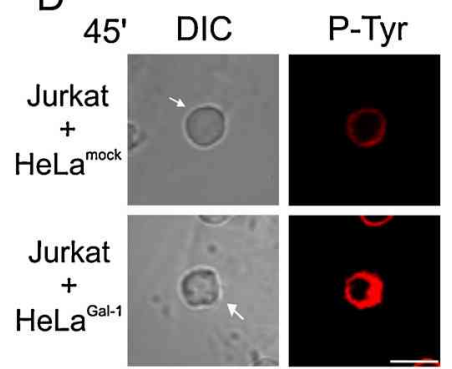

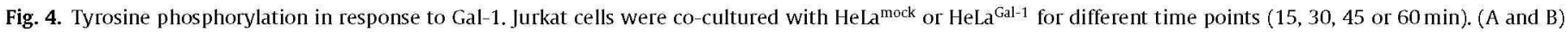

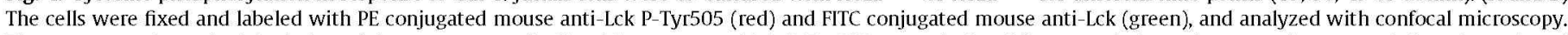

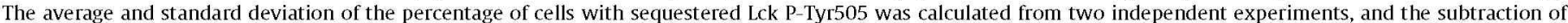

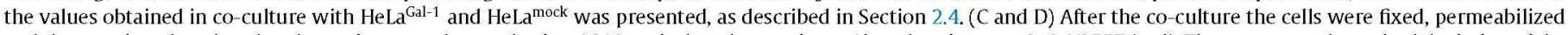

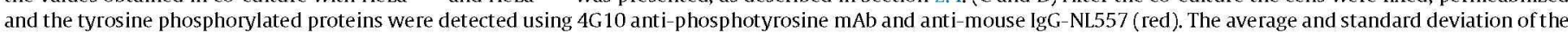

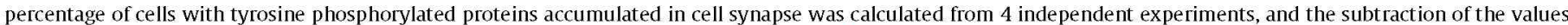

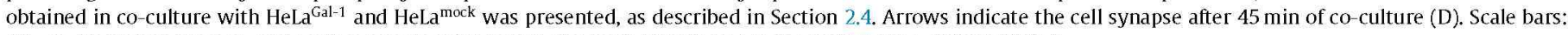
$10 \mu \mathrm{m}$. (For interpretation of the references to color in text, the reader is referred to the web version of this article.) 
A
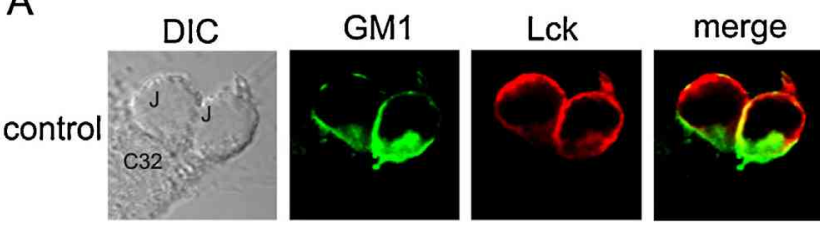

$\beta-C D$
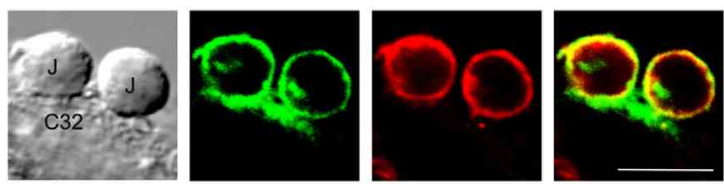

B
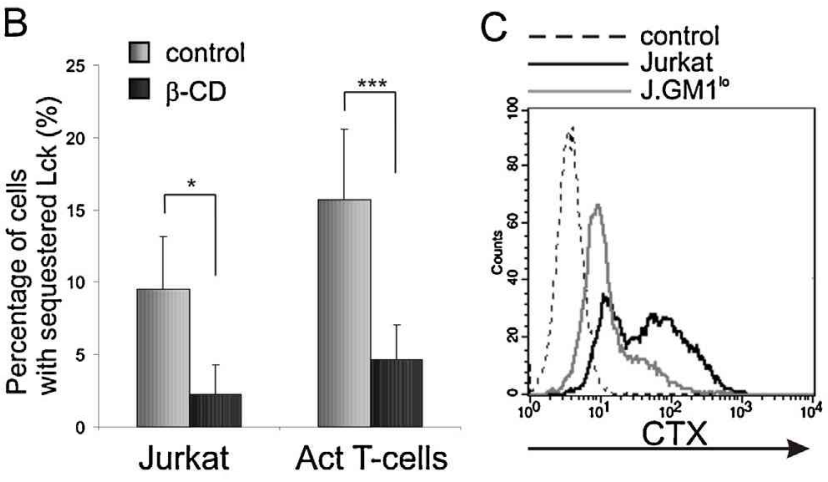

D

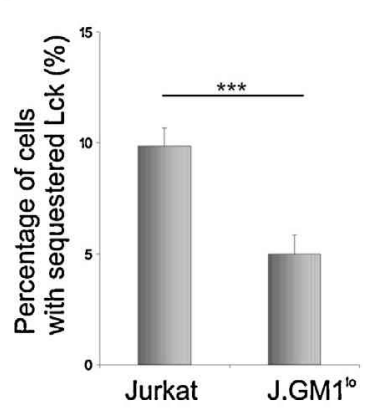

E

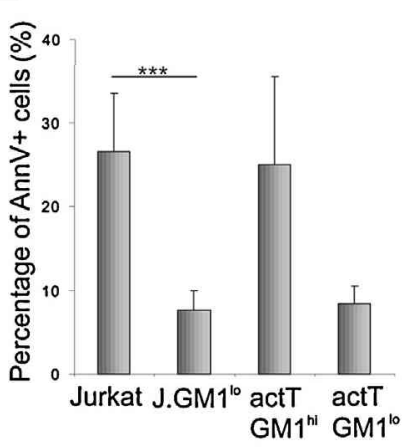

$\mathrm{F}$
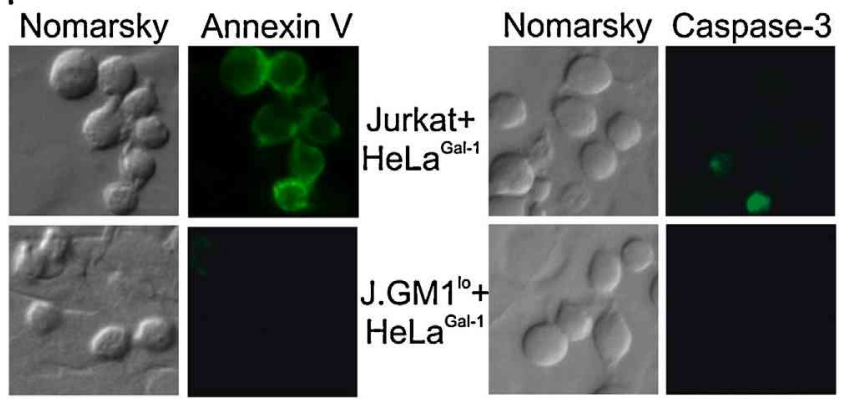

Fig. 5. Gal-1 induced apoptosis was dependent on GM1 expression of T-cells. (A) Jurkat cells were co-cultured with Gal-1 positive $\mathrm{C} 32$ cells for $1 \mathrm{~h}$ in the absence (control, upper row) or presence (lower row) of $10 \mathrm{mM} \beta$-CD. GM1 was detected by FITC conjugated CTX (green), Lck was labeled with mouse anti-Lck and anti-mouse IgG-NL557 (red), and then analyzed with confocal microscopy. Scale bar: $10 \mu \mathrm{m}$. (B) Jurkat cells or PHA-activated T-cells (actT) were co-cultured with HeLa ${ }^{\text {Gal-1 }}$ or HeLa ${ }^{\text {mock }}$ for $1 \mathrm{~h}$ in the presence or absence of $\beta-C D$. The percentage of cells with sequestered Lck was calculated from 5 (Jurkat) or 4 (T-cells) samples as described in Section 2.4. (C) The expression of GM1 on the cell surface was tested by flow cytometry after CTX-FITC binding to Jurkat cells (black line) or J.GM1 $1^{\text {lo }}$ (gray line), the control was left untreated (dotted line). (D) J.GM $1{ }^{10}$ or Jurkat cells were co-cultured with HeLa ${ }^{\mathrm{Gal}-1}$ or HeLa ${ }^{\text {mock }}$ for $1 \mathrm{~h}$, and then the sequestration of Lck was analyzed from 5 independent samples as described in Section 2.4. (E) Jurkat, J.GM1 ${ }^{10}$, the GM1 ${ }^{\text {lo }}$ or GM1 $1^{\text {hi }}$ subsets of activated human T-cells were co-cultured with HeLa ${ }^{\text {Gal-1 }}$ or HeLa ${ }^{\text {mock }}$ for $16 \mathrm{~h}$ and then reacted with Annexin V-AlexaFluor488. Apoptosis was detected by fluorescent microscopy, and calculated as described in Section 2.5 . apoptotic signal triggered by soluble Gal-1 in activated lymphocytes (Ion et al., 2006). Therefore we analyzed whether raft formation and interaction between Gal-1 and GM1 ganglioside regulated the Gal-1-triggered Lck membrane localization and eventually T-cell apoptosis. As presented on Fig. 5A, GM1 accumulated at the contact site between tumor and T-cells while Lck migrated to the opposite. In the presence of $\beta-C D$ both accumulation of GM1 (Fig. 5A) and segregation of Lck (Fig. 5A and B) failed either in Jurkat or activated T-cells (Fig. 5B), indicating that intact raft organization was crucial for this process. Since level of GM1ganglioside varies depending on the differentiation and activation state of the cells or in pathological situation (Wang et al., 2009), the question remains to be answered whether these changes affect the outcome of a signaling event. We found that Jurkat and activated T-cells are heterogeneous for GM1 expression (Fig. 5C and Supplementary Fig. 7A). Jurkat and activated T-cells expressing low level of GM1 were enriched by magnetic bead selection (J.GM110 , Fig. $5 \mathrm{C}$ and actT GM $1^{\text {lo }}$ Supplementary Fig. 7B) and co-cultured with HeLa cells. Segregation of Lck was significantly lower in J.GM1 ${ }^{\text {lo }}$ than in wild type Jurkat (Fig. 5D). In parallel, sensitivity to Gal-1 induced

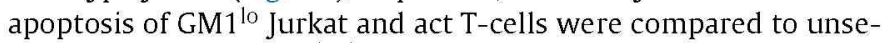
lected Jurkat and GM1 ${ }^{\text {high }}$ act T-cells, respectively. Reduction of cell surface level of GM1 resulted in diminution of apoptosis as detected with Annexin V-binding assay or caspase-3 activation in Jurkat (Fig. 5E and F) or activated T-cells (Fig. 5E).

\section{Discussion}

Apoptosis of T-lymphocytes is one of the hallmarks of the immunohomeostasis, including $\mathrm{T}$-cell differentiation and downregulation of effector functions. Among regulators of T-cell viability, Gal-1, a $\beta$-galactoside binding lectin has been emerged. Signaling pathway inducing death of activated T-cells and T-cell lines by Gal1 has recently been revealed (Hahn et al., 2004; Ion et al., 2006; Brandt et al., 2008). One of the most detailed description has been published in our laboratory (Ion et al,, 2005, 2006; Kovács-Sólyom et al., 2010; Blaskó et al., 2011), however even this failed to explain the earliest membrane events in context of the eventual apoptosis. Here we show that intimate cell contact between Gal-1 producing effector cells and activated $\mathrm{T}$ - or Jurkat target cells results in T-cell apoptosis which requires the early lateral segregation of nonreceptor tyrosine kinase, Lck, a central component of Gal-1 induced cell death.

Previous report by Pace et al. suggested that reorganization of certain molecules within the membrane might be an important step during Gal-1 triggered T-cell apoptosis since Gal-1 binding induced spatial redistribution of its binding receptors into specific microdomains (Pace et al., 1999). In their model CD45 clustered with CD3 and CD7 co-localized in small membrane patches with CD43. The major limitation of this system has been the usage of soluble recombinant human Gal-1 in high concentration (Pace et al., 1999), a condition which does not occur physiologically. Therefore we applied cell-derived Gal-1 in co-culture system (Kovács-Sólyom et al., 2010) to ensure the natural presentation of Gal-1 and hence modeling the in vivo milieu. Moreover co-culture method avoided the disadvantages raising from using recombinant protein, such as requirement of reducing agent for maintaining functionality and affecting cell viability of Gal-1 (Stowell et al., 2007, 2008), and controversial effects depending on the concentration of Gal-1 during

Student's $t$-test, ${ }^{*} p<0.05$, ${ }^{* * *} p<0.001$. (F) Jurkat or J.GM1 ${ }^{\text {lo }}$ were co-cultured with HeLa ${ }^{\text {Gal-1 }}$ for 16 (left panel) or $24 \mathrm{~h}$ (right panel) and apoptosis was detected with Annexin V-AlexaFluor488 (left panel) or rabbit anti-active caspase-3 and anti-rabbit Ig-FITC (right panel), then analyzed with fluorescent microscope. (For interpretation of the references to color in text, the reader is referred to the web version of this article.) 
the assays (Blaskó et al., 2011). It has to be noted that co-culture system has a disadvantage as well, namely that Gal-1 producing effector cells may express other galectins and unspecified factors which may affect the response of the target cells. Thus, the effects of the HeLa ${ }^{\text {mock }}$ (Gal-1 deficient), HeLa ${ }^{\text {mock }}$ covered with recombinant Gal-1 and Gal-1 transgenic counterpart, HeLa ${ }^{\mathrm{Gal}-1}$ cells were compared. The results clearly confirmed the specific contribution of Gal-1 to Lck lateral movement and, as previously showed, to apoptosis (Kovács-Sólyom et al., 2010), since Gal-1 treated HeLamock and HeLa ${ }^{\text {Gal-1 }}$ caused significantly higher Lck segregation and T-cell apoptosis than Gal-1 negative HeLamock. Moreover, the involvement of other galectin family members was excluded with lactose competition and real time PCR.

Encounter of T-cells with Gal-1 in a tumor cell-bound form induced a conducted rearrangement of membrane components, in which the cell-contact site determined the topological relations. After $30 \mathrm{~min}$ of cell-cell interaction Lck translocated to distal pole of the contact and p-Tyr505 Lck, the inactive form of the enzyme, strongly co-localized with the whole Lck pool. In contrast tyrosine phosphorylated proteins increasingly accumulated within the contact site up to $30 \mathrm{~min}$ of the cell-cell contact, then tyrosine phosphorylation signal declined. Receptor tyrosine phosphatase, CD45 played critical role in this process since specific inhibition or deficiency of CD45 prevented Lck sequestration and following apoptosis. As CD45 is one of the major regulators of Lck function by activating its kinase activity with dephosphorylating p-Tyr505 or maintaining Tyr 505 in dephosphorylated form (Sieh et al, 1993) it is conceivable that early activation of Lck within the cell contact zone is ensured by CD45. These results suggested the following serial of events: after interaction of effector Gal-1 presenting cells with T-cells, Lck promoted the tyrosine phosphorylation of signaling proteins accordingly our previous results (Ion et al., 2005, 2006; Kovács-Sólyom et al., 2010; Blaskó et al., 2011). After initiating the downstream processes, Lck left the signaling complex and was expelled to the distal region of T-cell membrane in an inactivated form. Sequestration of Lck from the cell synapse was sustained up to several h (data not shown), and the cells underwent apoptosis accompanied by phosphatidylserine externalization and caspase- 3 activation. The role of CD45 is worth for some discussion. Early studies were controversial in judging this question. First studies suggested that CD45 was the receptor for Gal-1 and it was indispensible in Gal1 induced apoptosis (Perillo et al., 1995). However, further studies failed to prove that CD45 is the apoptosis-mediating receptor for Gal-1 (Fajka-Boja et al., 2002; Pace et al., 2000). Finally the analysis of CD45 glycosylation clarified its role in Gal-1's cytotoxicity (Earl et al., 2010). It must be emphasized that all works, including our previous ones, used soluble Gal-1. The results presented here support the theory that in the case when Gal-1 acts as a cell- or extracellular matrix coupled protein, the pathways and signaling components leading to the eventual T-cell death may differ in some aspects from that induced by soluble, recombinant Gal-1.

Directed movement of membrane associated molecules largely depends on lipid components within the membrane. One of the determining ingredients is GM1-ganglioside which does not only play a role in raft generation but also regulates signal transduction. Activated T-cells show enhanced GM1 expression (Tani-ichi et al., 2005; Wang et al., 2009) suggesting an increase of the stimulatory pathways. Lowering the level of glycosphingolipids by using glucosylceramide synthase inhibitor attenuates TCR signaling, Tcell proliferation and IL-2 production (Zhu et al., 2011). Our recent results show that the integrity of membrane microdomains is essential during Gal-1 triggered T-cell apoptosis (Kovács-Sólyom et al., 2010). Accordingly, the GM1 composition of the cell membrane plays also a crucial role in response to Gal-1: Lck segregation is reduced and cells are less sensitive to Gal-1 induced apoptosis in T-cells with lower GM1 level compared to higher GM1 expressing T-cells. The importance of the GM1 level in T-cell membrane is also underscored by the observation that it is modified in pathological situations, such as in T-cells of diabetic NOD mice (Wu et al., 2011) or systemic lupus erythematosus (SLE) (Jury et al., 2004). Moreover, the altered GM1 composition along with reduced level of TCR $\zeta$-chain (Liossis et al., 1998) and Lck (Jury et al., 2003) leads to abnormal TCR signaling and dysfunction of SLE T-cells. Importantly, GM1 is a binding partner for Gal-1 (Kopitz et al., 1998; Wang et al., 2009), hence upon binding to Gal-1 it may participate in regulation of membrane movements ensuring the collection of extraand intracellular components of the membrane proximal signaling factors.

The current theory emphasize the regulatory role of lattice between galectins and cell surface glycans (Rabinovich et al., 2007; Garner and Baum, 2008; Grigorian et al., 2009; Ledeen et al., 2012). In parallel, our results show robust spatial remodeling of intracellular signaling elements in inner membrane leaflet upon encounter of T-cells with Gal-1 producing effector cells. When Gal-1 is presented by the producer/effector cell or the extracellular matrix, it defines a spatial distribution of membrane proteins in target T-cells, leading to sequestering of Lck apart from the cell-cell contact site, and switching the downstream signaling pathway from immune response to apoptosis.

\section{Conflict of interest}

The authors declare no conflict of interest.

\section{Acknowledgements}

We are grateful to Mrs. Andrea Gercsó for the excellent technical assistance, Dr. Ferhan Ayaydin and Dr. Imre Gombos for useful advices in confocal microscopy and image analysis, Edit Kotogány and Nóra Fehér for flow cytometric analysis, and Dr. Tamás Fehér for blood handling. This work was supported by grants from the Hungarian Scientific Research Fund [OTKA PD 75938, OTKA K 69047and NKTH-OTKA CK 78188] and TÁMOP-4.2.2.A$11 / 1 / K O N V-2012-0035$. RFB has been supported by the grant from the Hungarian Scientific Research Fund [OTKA PD 75938] and János Bolyai Research Fellowship of the Hungarian Academy of Sciences. The funders had no role in study design, data collection and analysis, decision to publish, or preparation of the manuscript.

\section{Appendix A. Supplementary data}

Supplementary material related to this article can be found, in the online version, at http://dx.doi.org/10.1016/ j.molimm.2013.10.010.

\section{References}

Blaskó, A., Fajka-Boja, R., Ion, G., Monostori, É., 2011. How does it act when soluble? Critical evaluation of mechanism of galectin- 1 induced T-cell apoptosis. Acta Biol. Hung, 62, 106-111.

Brandt, B., Büchse, T., Abou-Eladab, E.F., Tiedge, M., Krause, E., Jeschke, U., Walzel, H., 2008. Galectin-1 induced activation of the apoptotic death-receptor pathway in human Jurkat T lymphocytes. Histochem. Cell Biol. 129, 599-609.

Bromley, S.K., Burack, W.R., Johnson, K.G., Somersalo, K., Sims, T.N., Sumen, C, Davis, M.M., Shaw, A.S., Allen, P.M., Dustin, M.L., 2001. The immunological synapse. Annu. Rev. Immunol. 19, 375-396.

Burkhardt, J.K., Carrizosa, E., Shaffer, M.H., 2008. The actin cytoskeleton in T cell activation. Annu. Rev. Immunol. 26, 233-259.

Chitu, V., Demydenko, D., Tóth, G.K., Hegedüs, Z., Monostori, É., 1999. Conditions for permeabilization of cells used for intracellular tyrosine phosphorylation studies. BioTechniques 27, 435-437

Dykstra, M., Cherukuri, A., Pierce, S.K., 2001. Rafts and synapses in the spatial organization of immune cell signaling receptors. J. Leukoc. Biol. 70, 699-707. 
Earl, L.A., Bi, S., Baum, L.G., 2010. N- and O glycans modulate galectin-1 binding, CD45 signaling, and T cell death. J. Biol. Chem. 285, 2232-2244.

Fajka-Boja, R., Blaskó, A., Kovács-Sólyom, F., Szebeni, G.J., Tóth, G.K., Monostori, E., 2008. Co-localization of galectin-1 with GM1 ganglioside in the course of its clathrin- and raft-dependent endocytosis. Cell. Mol. Life Sci. 65, 2586-2593.

Fajka-Boja, R., Szemes, M., Ion, G., Légrádi, A., Caron, M., Monostori, E., 2002. Receptor tyrosine phos phatase, CD45 binds galectin-1 but does not mediate its apoptotic signal in T cell lines. Immunol. Lett. 82, 149-154.

Fouillit, M., Joubert-Caron, R., Poirier, F., Bourin, P., Monostori, E., Levi-Strauss, M., Raphael, M., Bladier, D., Caron, M., 2000. Regulation of CD45-induced signaling by galectin-1 in Burkitt lymphoma B cells. Glycobiology 10, 413-419.

Garner, O.B., Baum, L.G., 2008. Galectin-glycan lattices regulate cell-surface glyco protein organization and signalling. Biochem. Soc. Trans. 36, 1472-1477.

Grigorian, A., Torossian, S., Demetriou, M., 2009. T-cell growth, cell surface organization, and the galectin-glycoprotein lattice. Immunol. Rev. 230, $232-246$

Hahn, H.P., Pang, M., He, J., Hernandez, J.D., Yang, R.Y., Li, L.Y., Wang, X., Liu, F.T., Baum, L.G., 2004. Galectin-1 induces nuclear translocation of endonuclease $G$ in caspase- and cytochrome c-independent T cell death. Cell. Death Differ. 11, $1277-1286$.

He, J., Baum, L.G., 2004. Presentation of galectin- 1 by extracellular matrix triggers T cell death. J. Biol. Chem. 279, 4705-4712.

Ion, G., Fajka-Boja, R., Kovács, F., Szebeni, G., Gombos, I., Czibula, Á., Matkô, J., Monos tori, E., 2006. Acid sphingomyelinase mediated release of ceramide is essential to trigger the mitochondrial pathway of apoptosis by galectin-1. Cell. Signal. 18, $1887-1896$.

Ion, G., Fajka-Boja, R., Toth, G.K., Caron, M., Monostori, E., 2005. Role of p561ck and ZAP70-mediated tyrosine phosphorylation in galectin-1-induced cell death. Cell. Death Differ. 12, 1145-1147.

Ju, T., Xia, B., Aryal, R.P., Wang, W., Wang, Y., Ding, X., Mi, R., He, M., Cummings, R.D., 2011. A novel fluorescent assay for T-synthase activity. Glycobiology 21, $352-362$.

Jury, E.C., Kabouridis, P.S., Flores-Borja, F., Mageed, R.A., Isenberg, D.A., 2004. Altered lipid raft-associated signaling and ganglioside expression in T lymphocytes from patients with systemic lupus erythematosus. J. Clin. Invest. 113, 1176-1187.

Jury, E.C., Kabouridis, P.S., Abba, A., Mageed, R.A., Isenberg, D.A., 2003. Increased ubiquitination and reduced expression of LCK in T lymphocytes from patients with systemic lupus erythematosus. Arthritis Rheum. 48, 1343-1354.

Kawabuchi, M., Satomi, Y., Takao, T., Shimonishi, Y., Nada, S., Nagai, K., Tarakhovsky, A., Okada, M., 2000. Transmembrane phosphoprotein Cbp regulates the activities of Src-family tyrosine kinases. Nature 404, 999-1003.

Kopitz, J., von Reitzenstein, C., Burchert, M., Cantz, M., Gabius, H.J., 1998. Galectin-1 is a major receptor for ganglioside GM1, a product of the growth-controlling activity of a cell surface ganglioside sialidase, on human neuroblastoma cells in culture. J. Biol. Chem. 273, 11205-11211.

Kovács-Sólyom, F., Blaskó, A., Fajka-Boja, R., Katona, R.L., Végh, L., Novák, J., Szebeni, G.J., Krenács, L., Uher, F., Tubak, V., Kiss, R., Monostori, É., 2010. Mechanism of tumor cell-induced T-cell apoptosis mediated by galectin-1. Immunol. Lett. 127, $108-118$.

Ledeen, R.W., Wu, G., André, S., Bleich, D., Huet, G., Kaltner, H., Kopitz, J., Gabius, H.-J., 2012. Beyond glycoproteins as galectin counterreceptors: effector T cell growth control of tumors via ganglioside GM1. Ann. N.Y. Acad. Sci. 1253, 206-221.

Liossis, S.N., Ding, X.Z., Dennis, G.J., Tsokos, G.C., 1998. Altered pattern of TCR/CD3mediated protein-tyrosyl phosphorylation in T cells from patients with systemic lupus erythematosus. Deficient expression of the T cell receptor zeta chain. J. Clin. Invest. 101, 1448-1457.
Montixi, C Langlet, C, Bernard, AM Thimonier, J Dubois, C, Wurbel, MA Chauvin, J.P., Pierres, M., He, H.T., 1998. Engagement of T cell receptor triggers its recruitment to low-density detergent-insoluble membrane domains. EMBO J. $17,5334-5348$

Pace, K.E., Lee, C., Stewart, P.L., Baum, L.G., 1999. Restricted receptor segregation into membrane microdomains occurs on human T cells during apoptosis induced by galectin-1. J. Immunol. 163, 3801-3811.

Pace, K.E., Hahn, H.P., Pang, M., Nguyen, J.T., Baum, L.G., 2000. CD7 delivers a pro-apoptotic signal during galectin-1-induced T cell death. J. Immunol. 165 , 331-334.

Perillo, N.L., Pace, K.E., Seilhamer, J.J., Baum, L.G., 1995. Apoptosis of T cells mediated by galectin-1. Nature $378,736-739$.

Rabinovich, G.A., Toscano, M.A., Jackson, S.S., Vasta, G.R., 2007. Functions of cell surface galectin-glycoprotein lattices, Curr. Opin. Struct. Biol, 17, 513-520.

Rodgers, W., Rose, J.K., 1996. Exclusion of CD45 inhibits activity of p56lck associated with glycolipid-enriched membrane domains. J. Cell. Biol. 135, 1515-1523.

Rubinstein, N., Alvarez, M., Zwirner, N.W., Toscano, M.A., Ilarregui, J.M., Bravo, A. Mordoh, J., Fainboim, L., Podhajcer, O.L., Rabinovich, G.A., 2004. Targeted inhibition of galectin-1 gene expression in tumor cells results in heightened $T$ cell-mediated rejection: a potential mechanism of tumor-immune privilege. Cancer Cell. 5, 241-251.

Salmond, R.J., Filby, A., Qureshi, I., Caserta, S., Zamoyska, R., 2009. T-cell receptor proximal signaling via the Src-family kinases, Lck and Fyn, influences T-cell activation, differentiation, and tolerance. Immunol. Rev. 228, 9-22.

Sieh, M., Bolen, J.B., Weiss, A, 1993. CD45 specifically modulates binding of Lck to a phosphopeptide encompassing the negative regulatory tyrosine of Lck. EMBO J. $12,315-321$.

Stowell, S.R., Karmakar, S., Stowell, C. Dias-Baruffi, M. McEver, R.P. Cummings, R.D., 2007. Human galectin-1, -2 , and -4 induce surface exposure of phos phatidylserine in activated human neutrop hils but not in activated $\mathrm{T}$ cells. Blood $109,219-227$.

Stowell, S.R., Qian, Y., Karmakar, S., Koyama, N.S., Dias-Baruffi, M., Leffler, H. McEver, R.P., Cummings, R.D., 2008. Differential roles of galectin-1 and galectin3 in regulating leukocyte viability and cytokine secretion. J. Immunol. 180, 3091-3102.

Tani-ichi, S., Maruyama, K., Kondo, N., Nagafuku, M., Kabayama, K., Inokuchi, J., Shimada, Y., Ohno-Iwashita, Y., Yagita, H., Kawano, S., Kosugi, A., 2005. Structure and function of lipid rafts in human activated T cells. Int. Immunol. 17, 749-758.

Tuosto, L., Parolini, I., Schröder, S., Sargiacomo, M., Lanzavecchia, A., Viola, A., 2001. Organization of plasma membrane functional rafts upon T cell activation. Eur. J. Immunol. 31, 345-349.

Walzel, H., Schulz, U., Neels, P., Brock, J., 1999. Galectin-1, a natural ligand for the receptor-type protein tyrosine phosphatase CD45. Immunol. Lett. 67, 193-202.

Wang, J., Lu, Z.-H., Gabius, H.-J., Rohowsky-Kochan, C., Ledeen, R.W., Wu, G., 2009. Cross-linking of GM1 ganglioside by galectin-1 mediates regulatory $\mathrm{T}$ cell activity involving TRPC5 channel activation: possible role in suppressing experimental autoimmune encephalomyelitis. J. Immunol. 182, 4036-4045.

Wu, G., Lu, Z.-H., Gabius, H.-J., Ledeen, R.W., Bleich, D., 2011. Ganglioside GM1 deficiency in effector $\mathrm{T}$ cells from NOD mice induces resistance to regulatory T-cell suppression. Diabetes $60,2341-2349$.

Zhu, Y., Gumlaw, N., Karman, J., Zhao, H., Zhang, J., Jiang, J.-L., Maniatis, P., Edling, A., Chuang, W.-L., Siegel, C., Shayman, J.A., Kaplan, J., Jiang, C., Cheng, S.H., 2011. Lowering glycosphingolipid levels in CD4+ T cells attenuates $\mathrm{T}$ cell receptor signaling, cytokine production, and differentiation to the Th17 lineage. J. Biol. Chem. 286, 14787-14794 